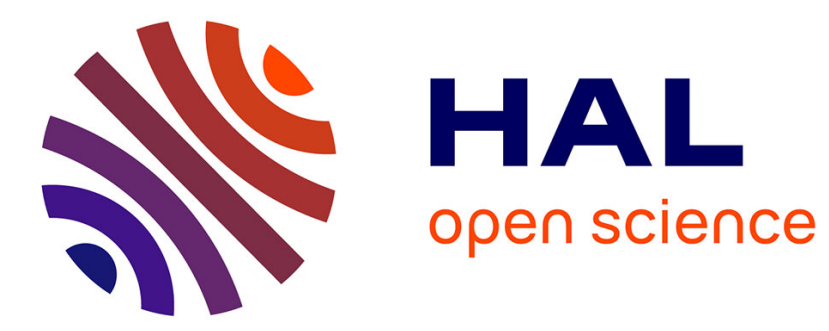

\title{
Viscosity and Morphology of Water-in-Water Emulsions: The Effect of Different Biopolymer Stabilizers
}

\author{
Lingsam Tea, Taco Nicolaï, Lazhar Benyahia, Frédéric F. Renou
}

\section{To cite this version:}

Lingsam Tea, Taco Nicolaï, Lazhar Benyahia, Frédéric F. Renou. Viscosity and Morphology of Waterin-Water Emulsions: The Effect of Different Biopolymer Stabilizers. Macromolecules, 2020, 53 (10), pp.3914-3922. 10.1021/acs.macromol.0c00204 . hal-03026664

\section{HAL Id: hal-03026664 https://hal.science/hal-03026664}

Submitted on 3 Dec 2020

HAL is a multi-disciplinary open access archive for the deposit and dissemination of scientific research documents, whether they are published or not. The documents may come from teaching and research institutions in France or abroad, or from public or private research centers.
L'archive ouverte pluridisciplinaire HAL, est destinée au dépôt et à la diffusion de documents scientifiques de niveau recherche, publiés ou non, émanant des établissements d'enseignement et de recherche français ou étrangers, des laboratoires publics ou privés. 


\section{Viscosity and morphology of water-in-water 2 emulsions: the effect of different biopolymer 3 stabilizers.}

4

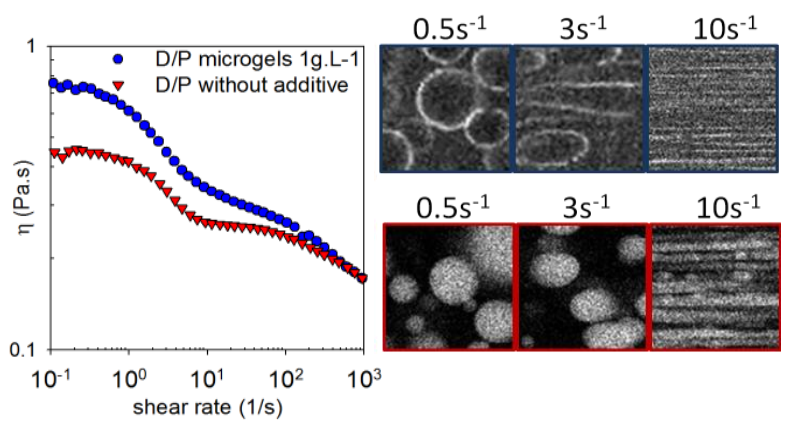

12 


\section{Abstract}

15 Water-in-water (W/W) emulsions show a characteristic decrease of the viscosity with increasing

16 shear rate that is well described by equations proposed in the literature. Confocal laser scanning

17 microscopy images showed that the decrease of the viscosity was caused by deformation and

18 alignment of the dispersed droplets followed by string formation. W/W emulsions stabilized by

19 addition of polysaccharides or protein microgels still form strings when sheared with the

20 particles remaining at the interface. After cessation of the flow the strings break up into small

21 droplets and the presence of stabilizing particles inhibits their coalescence. It is shown how the

22 viscosity and the microstructure depend on the initial droplet size, the interfacial tension, the

23 viscosity of the two phases and the concentration of the stabilizing polymers.

24 KEYWORDS Emulsion; viscosity; aqueous two phase; mixture, structure; string

26 I. Introduction

Water-in-water (W/W) emulsions are formed by mixing two aqueous solutions of

29 incompatible polymers ${ }^{1}$. Such emulsions are different from oil-in-water $(\mathrm{O} / \mathrm{W})$ emulsions in that

30 their interfacial tension is orders of magnitude lower and their interface is broader. As a result,

31 stabilization of W/W emulsions cannot be achieved by molecular surfactants. However, they can

32 be stabilized by adding particles, which has received considerable attention in recent years, see

33 for reviews ref. ${ }^{2-4}$. Recently, we reported that they can also in some cases be stabilized by linear

34 polyelectrolytes ${ }^{5}$. The fact that stable W/W emulsions can be obtained opens the possibility to 
35 use them for application in areas such as nutrition, cosmetics or pharmacy. It is therefore of 36 interest to investigate their behavior under flow.

37 Taylor ${ }^{6}$ was the first to propose an equation to describe the zero shear rate viscosity $\left(\eta_{0}\right)$

38 of emulsions in general as a function of the volume fraction of the dispersed phase $(\phi)$ and the

39 viscosity ratio $(K)$ between that of the dispersed $\left(\eta_{d}\right)$ and the continuous $\left(\eta_{c}\right)$ phases:

40

$$
\eta_{0}=\eta_{c}\left[1+\phi \frac{5 K+2}{2 K+2}\right]
$$

43 This equation is valid only for dilute emulsions of incompressible liquids and does not include

44 the effect of the shear rate $(\dot{\gamma})$, the radius of the droplets $(\mathrm{R})$ or the interfacial tension $(\Gamma)$. Under 45 shear, the droplets can deform and align which leads to a reduction of the viscosity. These effects 46 were taken into consideration by $\operatorname{Kroy}^{7}$ (eq. 2) and by Frankel and $\operatorname{Acrivos}^{8}$ (eq.3):

$$
\eta=\frac{\eta_{c}}{1+(\tau \dot{\gamma})^{2}}\left[\frac{2 K+2+3 \Phi(K+0.4)}{2 K+2-2 \Phi(K+0.4)}+\frac{2 K+3+3 \Phi(K-1)}{2 K+3-2 \Phi(K-1)}(\tau \dot{\gamma})^{2}\right]
$$

$$
\eta=\frac{\eta_{c}}{1+(\tau \dot{\gamma})^{2}}\left[1+\Phi \frac{5 K+2}{2 K+2}+\left(1+\Phi \frac{5(K-1)}{2 K+3}\right)(\tau \dot{\gamma})^{2}\right]
$$

with

$$
\tau=\frac{\eta_{c} R}{\Gamma} \frac{(19 K+16)(2 K+3-2 \Phi(K-1))}{40(K+1)-8 \Phi(5 K+2)}
$$

55 Here $\tau$ is the relaxation time of the droplets. 
It is known that isolated droplets break above a critical shear rate that depends on the

57 capillary number $\left(\mathrm{Ca}=\eta_{c} \dot{\gamma} R / \Gamma\right)$ and $\mathrm{K}^{9}$. However, studies on blends of incompatible synthetic

58 polymers have shown that at higher dispersed volume fractions the deformed droplets align in

59 the flow direction followed by rupture and coalescence to form strings above a critical shear rate

60 that become thinner with increasing shear rate ${ }^{10-13}$. After cessation of the flow the strands quickly

61 break up into small droplets due to Rayleigh instabilities.

62 W/W emulsions formed by mixing incompatible biopolymers have been much less

63 investigated ${ }^{14-17}$. Capron et al. ${ }^{15}$ investigated emulsions consisting of droplets of a phase rich in

64 alginate dispersed in a continuous caseinate rich phase and vice versa. They found that the shear

65 dependence of the viscosity could be well described by eq. 2 . These authors did not study the

66 morphology of the emulsions under shear and assumed that the dispersed droplets broke up

67 above a critical shear rate. Wolf and Frith $^{17}$ studied the shear viscosity of W/W emulsions

68 formed by mixtures of gelatin and dextran. They found that the viscosity at low shear rates and

69 low volume fractions of the dispersed phase could be well described by an equation proposed by

70 Choi and Schowalter ${ }^{18}$, see eq.5, whereas the viscosity at high shear rates could be well

71 described by a simple logarithmic mixing law over the whole range of volume fractions, see

72 eq.6:

73

$74 \quad \eta=\eta_{c}\left[\frac{1+h_{1} h_{2} \dot{\gamma}^{2}}{1+h_{2}^{2} \dot{\gamma}^{2}}\right]$

75 with

$76 h_{1}=\tau\left[1+\Phi \frac{5(19 K+16)}{4(K+1)(2 K+3)}\right]$ and $h_{2}=\tau\left[1+\Phi \frac{3(19 K+16)}{4(K+1)(2 K+3)}\right]$

77

$78 \log \left(\eta_{c}\right)=\phi \log \left(\eta_{d}\right)+(1-\phi) \log \left(\eta_{c}\right)$ 
80 They observed that strings were formed above a critical shear rate that depended on the volume

81 fraction and the viscosity ratio. The effect of shear on the morphology of $\mathrm{W} / \mathrm{W}$ emulsions formed

82 by the same biopolymer mixtures was investigated in more detail by Tromp and De Hoog ${ }^{14}$.

83 These authors found that at a fixed shear rate the strings coalesce laterally with time into thicker

84 strands and eventually form bands.

85 Here we present a more extensive and detailed investigation of the viscosity and the 86 morphology of model W/W emulsions formed by mixtures of poly(ethylene oxide) (PEO) and

87 dextran as a function of the shear rate. The shear rate dependence of the viscosity will be

88 compared with the theoretical predictions mentioned above. The morphology was studied as a

89 function of the shear rate utilizing confocal laser scanning microscopy (CLSM) combined with a

90 specially designed shear device. The effect of the volume fraction and the interfacial tension was

91 investigated. Furthermore, we compare the results obtained for unstable W/W emulsions with

92 those obtained for W/W emulsions stabilized by the presence of different types of

93 polysaccharides or by protein microgels. The effect of shear on the viscosity and morphology of

94 stable W/W emulsions has not been reported before in the literature.

96 II. Materials and methods

97

\section{Sample preparation}

99 Dextran and PEO with weight average molar masses $\mathrm{M}_{\mathrm{w}}=1.6 \times 10^{5} \mathrm{~g} \cdot \mathrm{mol}^{-1}$ and $\mathrm{M}_{\mathrm{w}}=2 \times$

$10010^{5}$ g.mol ${ }^{-1}$, respectively, were purchased from Sigma-Aldrich. The powders were dissolved in 
101 ultra-pure water (MilliQ). The PEO sample contained a very small amount of silica particles that 102 were removed by centrifugation at $5 \times 10^{4} \mathrm{~g}$ for $4 \mathrm{~h} 30$. Chitosan is a cationic polysaccharide with a $103 \mathrm{pKa} \approx 6.5^{19}$. The chitosan sample used for this study was purchased from Sigma-Aldrich (batch 104 STBG5137V) and had a degree of acetylation of $25 \%$ as determined by NMR. Diethyl 105 aminoethyl dextran (DEAED) is a cationic polysaccharide obtained by functionalizing dextran 106 with diethyl aminoethyl groups that are positively charged in a wide $\mathrm{pH}$ range $(\mathrm{pKa} \approx 9.5)^{20}$. The 107 sample used for this study was purchased from Sigma-Aldrich and contained $80 \%$ functionalized 108 sugar units. Stock solutions of chitosan and DEAED were prepared in MilliQ water by stirring 109 overnight. א-Carrageenan (KC) was purchased from Sigma-Aldrich. The weight average molar 110 mass $\left(\mathrm{M}_{\mathrm{w}}\right)$ and $\mathrm{z}$-average hydrodynamic radii $\left(\mathrm{R}_{\mathrm{h}}\right)$ were determined by light scattering 111 techniques 5 yielding: $\mathrm{M}_{\mathrm{w}}=3.4 \times 10^{5} \mathrm{~g} / \mathrm{L}$ and $\mathrm{R}_{\mathrm{h}}=58 \mathrm{~nm}$ for chitosan, $\mathrm{M}_{\mathrm{w}}=8 \times 10^{5} \mathrm{~g} / \mathrm{mol}$ and $\mathrm{R}_{\mathrm{h}}=$ $11239 \mathrm{~nm}$ for DEAED and $\mathrm{M}_{\mathrm{w}}=3.5 \times 10^{5} \mathrm{~g} / \mathrm{mol}$ and $\mathrm{R}_{\mathrm{h}}=60 \mathrm{~nm}$ for KC. Protein microgel particles 113 were prepared by heating solutions at $\mathrm{pH} 6.1$ of whey protein isolate (WPI) purchased from 114 Lactalis (Laval, France) at a concentration of $40 \mathrm{~g} / \mathrm{L} . \mathrm{R}_{\mathrm{h}}$ of the microgels was measured by 115 dynamic light scattering as described elsewhere ${ }^{21}$ and found to be $140 \mathrm{~nm}$.

116 The emulsions were prepared by mixing the required amounts of each biopolymer 117 solution using a vortex mixer. No effect of the mixing order or the mixing speed was seen on the 118 morphology of the emulsions. We suggest that sufficient turbulent flow leads to complete 119 homogeneous mixture of all components. Same results were obtained by dissolving the polymer 120 in the PEO phase or dextran phase first. The $\mathrm{pH}$ was set to the desired values by addition of a 121 small amount of $\mathrm{NaOH}(0.1 \mathrm{M})$ or $\mathrm{HCl}(0.1 \mathrm{M})$. The behavior of unstable emulsions, pure or in 122 the presence of $\mathrm{KC}$, was independent of the $\mathrm{pH}$. Emulsions in the presence of protein microgels 123 were stable at $\mathrm{pH}$ 7.0. Those in the presence of DEAED or chitosan were most stable at $\mathrm{pH}$ 5.0. 


\section{Interfacial tension}

126 The interfacial tension of $\mathrm{W} / \mathrm{W}$ emulsions is very low and is very difficult to determine

127 accurately. Here we determined $\Gamma$ at $20^{\circ} \mathrm{C}$ by analyzing the shape of the interface near a wall in 128 terms of the capillary length $\left(1_{c}\right)^{22}$ :

$$
\frac{x(z)}{l_{c}}=\operatorname{arccosh}\left(\frac{2 l_{c}}{z}\right)-\operatorname{arccosh}\left(\frac{2 l_{c}}{h}\right)-\sqrt{4-\frac{z^{2}}{l_{c}^{2}}}+\sqrt{4-\frac{h^{2}}{l_{c}^{2}}}
$$

132 where $\mathrm{x}$ is the distance to a vertical wall, $\mathrm{z}$ is the height of the interface with respect to that far

133 from the wall and $\mathrm{h}$ is the height at the wall. From the capillary length and the density difference

134 between the two phases $(\Delta \rho)$ the interfacial tension can be calculated:

$$
l_{c}=\sqrt{\frac{\Gamma}{\Delta \rho g}}
$$

138 with $\mathrm{g}$ the gravitational constant. $\Delta \rho=50 \mathrm{~kg} / \mathrm{mm}^{3}$ was measured within $\pm 1 \mathrm{~kg} / \mathrm{mm}$ with a Metler

139 Toledo DM50 oscillating U-tube densimeter and was found within the experimental error to be

140 the same with and without added polysaccharides The two phases were gently placed on top of

141 each other in polystyrene cells after which the cells were centrifuged to remove air bubbles and

142 to ensure full separation of the phases. The interface was imaged with a Nikon camera and the

143 digitized interface was fitted to eq. 7, see figure S1 of the supplementary information. 
147 TA instruments) using a cone plate geometry (radius $40 \mathrm{~mm}$, angle $2^{\circ}$ ). The emulsion was placed

148 in the geometry and presheared at $10^{3} \mathrm{~s}^{-1}$ during $30 \mathrm{~s}$, but longer preshear was found not to affect

149 the results. Subsequently, the viscosity was measured while decreasing the shear rate from 1000 $150 \mathrm{~s}^{-1}$ to $0.01 \mathrm{~s}^{-1}$ and then increasing $\dot{\gamma}$ back to $1000 \mathrm{~s}^{-1}$. We have applied fast shear rate ramps 151 taking only 2 min in order to avoid coalescence of the dispersed droplets, which is favored by 152 flow at low shear rates. At these conditions, the same viscosities were measured within $10 \%$ 153 when repeating shear ramps with the same sample or with different samples of the same 154 emulsion. The effect of the duration of the ramp is shown in Fig. S2a of the supplementary 155 information for unstabilized emulsions. No difference was found when the shear ramp took 1 or $1562 \mathrm{~min}$, but when the shear rate was increased more slowly the decrease of the viscosity started at 157 smaller $\dot{\gamma}$ and reached a higher plateau. Both at very low $\left(\dot{\gamma}<0.1 \mathrm{~s}^{-1}\right)$ and very high shear rates 158 the results were not significantly dependent on the duration of the shear ramp. The effect of the 159 duration of the shear rate ramp was weaker for emulsions in the presence of chitosan, see Fig. 160 S2b of the supplementary information, which might be expected as chitosan inhibited 161 coalescence. The temperature was controlled by a Peltier to within $\pm 0.1^{\circ} \mathrm{C}$.

\section{Confocal laser scanning microscopy}

164 Images were obtained with a Zeiss LSM800 CLSM microscope (Carl Zeiss Microscopy $165 \mathrm{GmbH}$, Germany) with two different objectives: HC $\times$ PL APO $10 \times(\mathrm{NA}=1.2)$ and a water 166 immersion $\mathrm{HC} \times \mathrm{PL}$ APO $25 \times(\mathrm{NA}=0.7)$. Images were taken at a rate of 3 frames per second. It 167 was shown elsewhere that the layer of chitosan or DEAED is very difficult to visualize using 168 labeled polysaccharides. However, the layer of labeled protein microgel could be easily 
169 observed. Therefore, in most emulsions only the dextran phase was visualized by adding a small

170 amount of dextran labeled with FITC. In emulsions with protein microgels a small amount of

171 rhodamine B was added that binds spontaneously to the proteins. The droplets can nevertheless

172 still be observed because excess rhodamine B not bound to the microgels was not distributed

173 equally between the two phases. FITC was excited at $488 \mathrm{~nm}$, whereas rhodamine B was excited

174 at $561 \mathrm{~nm}$. The morphology of the emulsions under shear was visualized using a RheOptiCAD®

175 device (CAD Instruments, Les Essarts-le-Roi, France), consisting of two parallel plates

176 translating in opposite direction. The device can be used with different inversed microscopes and

177 was used here in combination with the CLSM microscope. For a detailed description of the

178 device see ref. ${ }^{23}$. The time during which the samples could be sheared depended on the gap and

179 the shear rate and was only a few seconds at the highest shear rates used here. The gap was set at 180 values between $500 \mu \mathrm{m}$ and $200 \mu \mathrm{m}$ and the emulsions were sheared at different rates between

1810.5 and $120 \mathrm{~s}^{-1}$ after a preshear at $10 \mathrm{~s}^{-1}$ in order to start at each shear rate with approximately the 182 same droplet size distribution.

\section{Results and discussion}

In first instance, we investigated emulsions consisting of droplets of the PEO phase in a

187 continuous dextran phase $(\mathrm{P} / \mathrm{D})$ and vice versa $(\mathrm{D} / \mathrm{P})$. Emulsions with different volume fractions 188 of the dispersed phase were prepared at the same tie-line where phase separation was almost 189 complete with $161 \mathrm{~g} / \mathrm{L}$ dextran in one phase and $80.5 \mathrm{~g} / \mathrm{L}$ PEO in the other phase. The emulsions 190 where homogenized with a vortex mixer and immediately placed in the apparatus. Subsequently, 191 the emulsions were presheared at $10 \mathrm{~s}^{-1}$ during $5 \mathrm{~s}$. Longer preshear did not affect the 
192 morphology significantly. Figure 1 shows the morphology of the emulsions at rest immediately

193 after being presheared at different phase volume fractions. The droplet size increased with

194 increasing volume fraction of the dispersed phase and phase inversion is observed when the PEO

195 phase volume fraction approached $50 \%$.
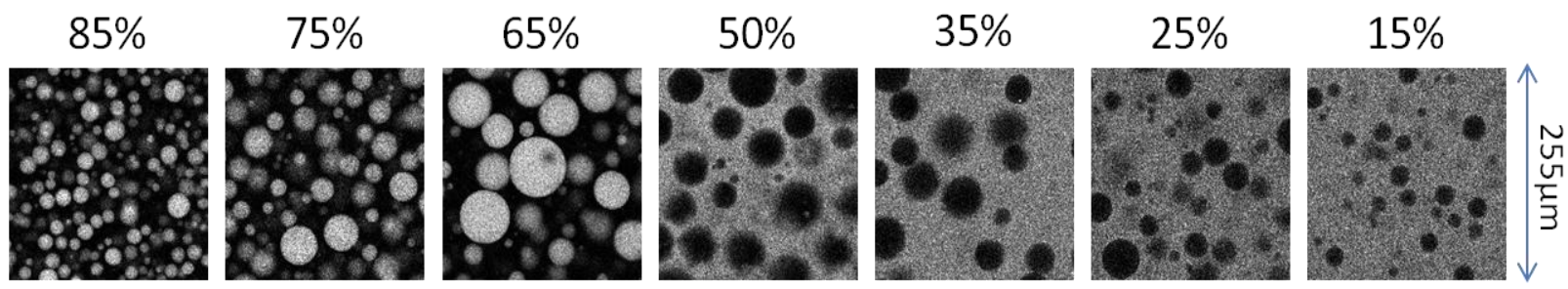

197 Figure 1. CLSM images obtained just after mixing of P-D emulsions at different PEO phase

198 volume fractions indicated in the figure. The labeled dextran phase is indicated in white.

\section{Viscosity}

In figure 2 the viscosity is shown as a function of the shear rate for a $\mathrm{P} / \mathrm{D}$ and a $\mathrm{D} / \mathrm{P}$ emulsion at $\phi=0.25$. The viscosity was found to decrease with increasing shear rate, but there was a distinct difference between results obtained during increasing and decreasing $\dot{\gamma}$. The same

204 results were obtained when the shear rate ramps were repeated implying that there was no 205 significant loss of material caused by shearing at high rates. Results obtained for the corresponding pure PEO and dextran phases are also shown in figure 2 . The viscosity of the PEO

207 phase was higher than that of the dextran phase and shows an effect of shear thinning for $\dot{\gamma}>30$

$208 \mathrm{~s}^{-1}$. The comparison shows that $\eta_{0}$ was significantly larger for the emulsions than for the 209 continuous phase. The effect of shear thinning was similar for the $\mathrm{D} / \mathrm{P}$ emulsion and the pure 210 PEO phase. Shear thinning for the P/D emulsion was much weaker than for the $\mathrm{D} / \mathrm{P}$ emulsion, 211 but it was stronger than that for the pure dextran phase. We may conclude that shear thinning of 
212 the continuous phase is more important, but that shear thinning of the dispersed phase also

213 influences the viscosity of the emulsions.

214

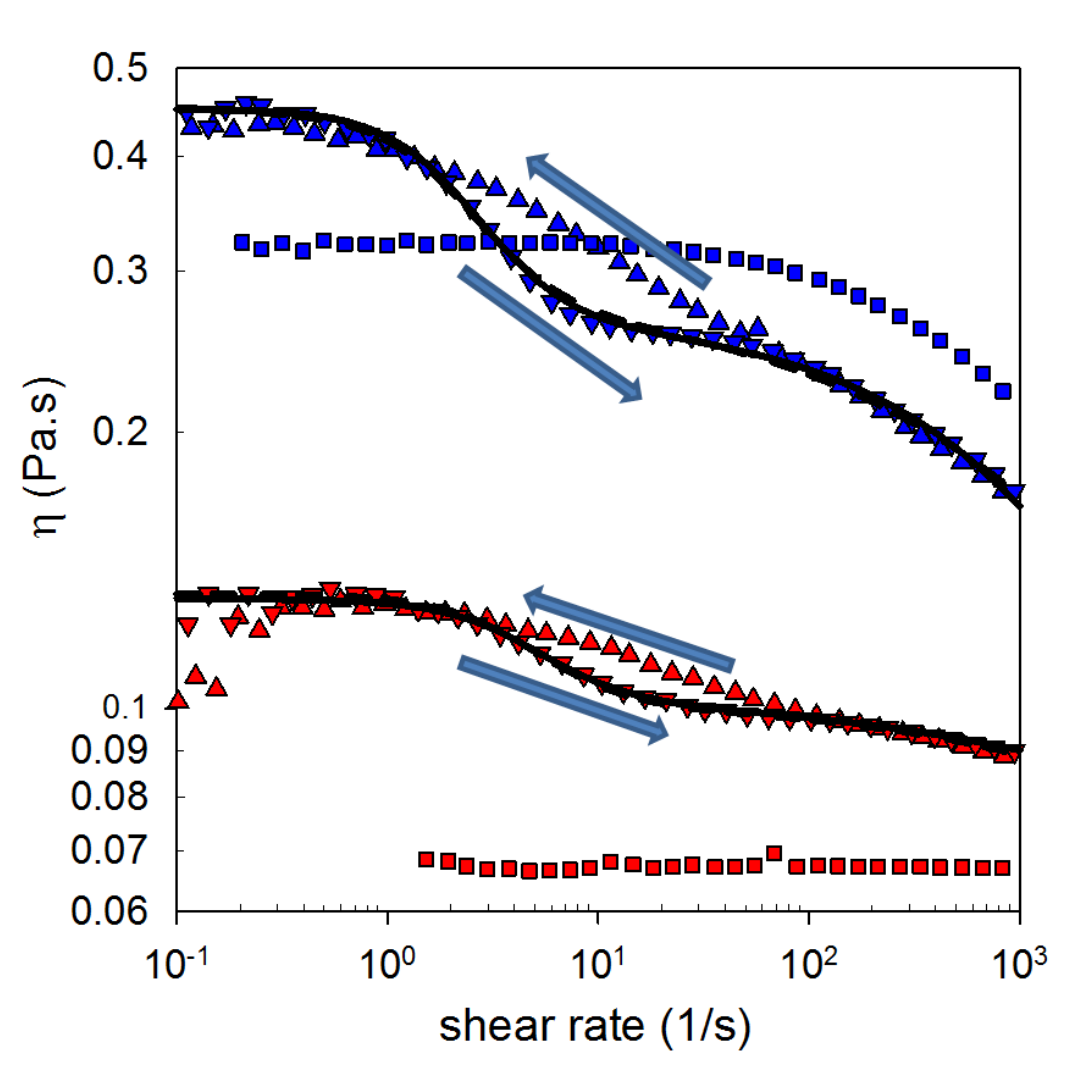

216 Figure 2. Viscosity as a function of the shear rate for emulsions of dextran in PEO (D/P) (blue

217 triangles) and PEO in dextran (P/D) (red triangles) at $\phi=0.25$. The total duration of the shear

218 rate ramp was 2 min. For comparison the viscosities of the pure PEO (blue squares) and dextran

219 (red squares) phases are included. The solid and dashed lines represent fits of $\eta$ during increasing

220 shear rate to eqs 2 and 3, respectively.

Figure 3 shows the viscosity as a function of increasing shear rate for emulsions at 223 different phase volume fractions. At all volume fractions hysteresis similar to that shown in 
224 figure 2 was observed for decreasing shear rate, which was not shown in figure 3 for clarity. The 225 zero-shear viscosity $\left(\eta_{0}\right)$ increased with increasing volume fraction of the more viscous PEO 226 phase $\left(\phi_{\mathrm{PEO}}\right)$ until it reached a maximum at approximately $75 \%$ and then decreased to that of the 227 pure PEO phase, see figure 4. The high shear rate viscosity $\left(\eta_{\propto}\right)$ was determined at 228 approximately $20 \mathrm{~s}^{-1}$, i.e. at the high frequency plateau where the effect of shear thinning was 229 still negligible. $\eta_{\propto}$ was found to increase progressively with increasing $\phi_{\mathrm{PEO}}$, see figure 4 .

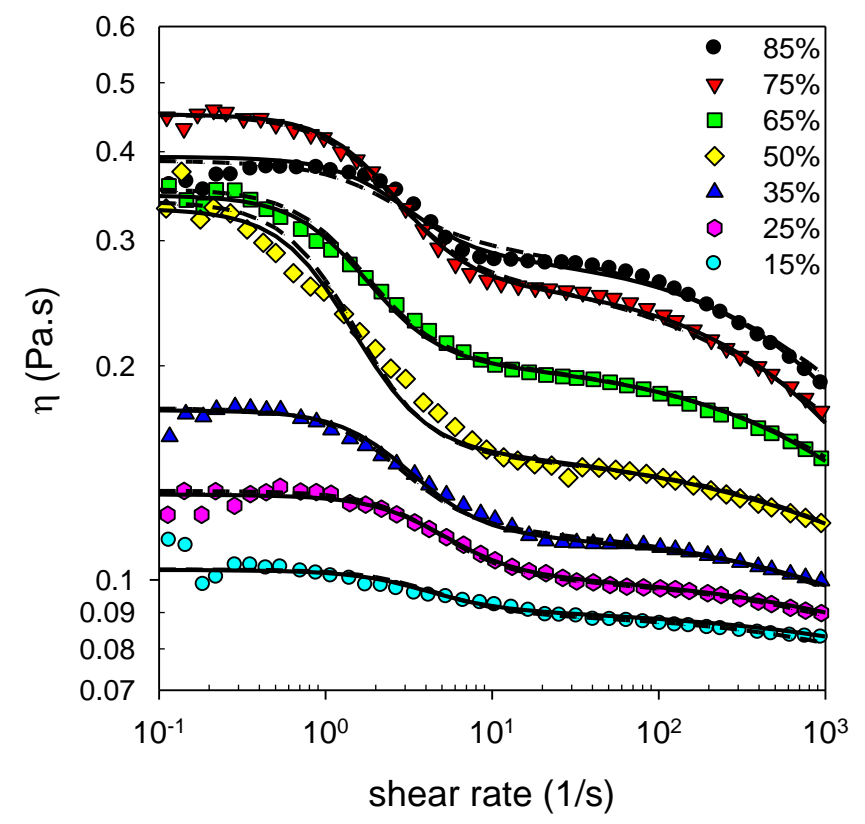

231 Figure 3. Viscosity as a function of increasing shear rate for P-D emulsions at different volume 232 fractions of the PEO phase as indicated in the figure. The solid and dashed lines represent fits to 233 eqs 2 and 3 , respectively. The dependence of $\eta_{0}$ and $\eta_{\infty}$ on $\phi_{\mathrm{PEO}}$ is compared in figure 4 with that calculated using 236 eqs 2 and 3, which describe the data reasonably well at low volume fractions of the dispersed 237 phase. The values of $\eta_{0}$ calculated using eq. 3 are equal to those proposed by Taylor (eq.1). Choi 
238 and Schowalter ${ }^{18}$ also proposed an equation for $\eta_{0}$ as a function of the volume fraction (see eq.5),

239 but the predicted values were much larger than the experimental data even at low volume

240 fractions of the dispersed phase. A similar discrepancy was reported for the W/W emulsions

241 studied by Kroy et al. However, Wolf and Frith ${ }^{17}$ reported that the viscosity at low shear rate was

242 well described by the eq. 5 for dispersed volume fractions lower than $20 \%$. The high shear rate

243 viscosities were also reasonably well described by eqs 2 and 3 at low dispersed volume fractions,

244 but a simple logarithmic mixing rule (eq.5) describes the data somewhat better over the whole

245 range of volume fractions. This was also the case for the $\mathrm{W} / \mathrm{W}$ emulsions studied by Wolf et

246 Frith $^{17}$. It is perhaps not surprising that the results at high shear rates do not correspond to the

247 theoretical predictions, because, as we will show below, strands are formed, which is not

248 considered in the theory. The discrepancy of $\eta_{0}$ at larger dispersed volume fractions shows that

249 the effect of interaction between the dispersed droplets was not correctly considered in the 250 theory. 


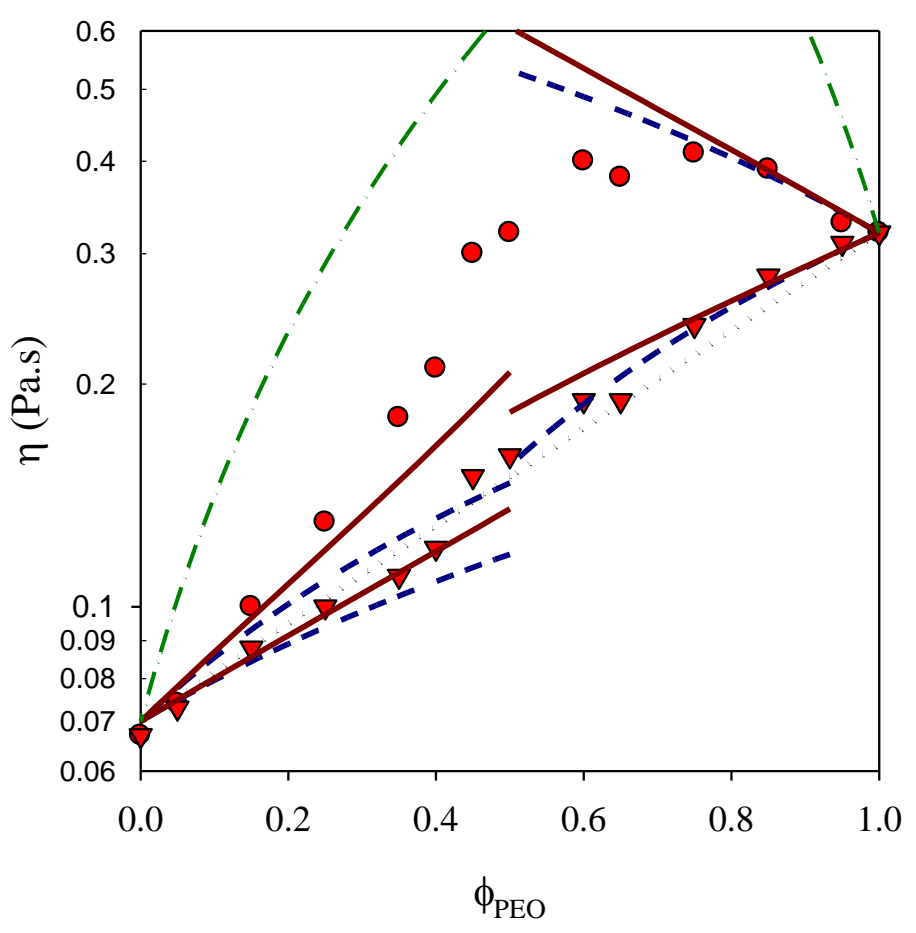

252 Figure 4. High (circle) and low (triangle) plateau viscosities as a function of the volume fraction 253 of the PEO phase. The solid, dashed and dashed-dotted lines indicate theoretical predictions 254 given by eqs 2,3 and 5, respectively. The dotted line represents a logarithmic mixing rule (eq. 5).

The shear rate dependent viscosity was compared to the theoretical predictions given by 257 eqs 2 and 3, see solid and dashed lines in figures 2 and 3. It was found that both equations well 258 described the viscosity with increasing shear rate, but not with decreasing shear rate. In fact, the 259 fit results obtained by eqs 2 and 3 practically overlap. Only for emulsions with equal phase 260 volumes was the decrease of $\eta$ with increasing shear rate more gradual than predicted by eqs 2 261 and 3. We note that it was necessary to include the effect of shear thinning on the viscosity of the 262 PEO phase in order to fit the data at high shear rates. It appears that the three fit parameters of 263 eqs 2 and $3\left(\eta_{\mathrm{c}}, \eta_{\mathrm{d}}\right.$ and $\left.\tau\right)$ are enough to describe the viscosity well as a function of increasing 
264 shear rate in spite of the fact that the theory does not consider strand formation at high shear 265 rates. The real test of the theory is whether the values obtained from the fits are physically 266 realistic.

267 In most cases there was little difference between the values of $\eta_{c}, \eta_{d}$ and $\tau$ obtained from 268 fits to eq 2 or eq 3, see figure 5. $\eta_{\mathrm{c}}$ was close to that of the pure PEO for $\phi_{\mathrm{PEO}}>75 \%$ and close to 269 that of the dextran phase for $\phi_{\mathrm{PEO}} \leq 25 \%$, see figure $5 \mathrm{a}$. At intermediate volume fractions, $\eta_{\mathrm{c}}$ was 270 intermediate between that of the pure phases. We believe that the agreement at $\phi_{\mathrm{PEO}}=50 \%$ 271 between $\eta_{c}$ obtained from a fit to eq. 2 and the viscosity of the pure dextran phase is fortuitous.

272 The values of $\eta_{d}$ obtained from fits were in all cases intermediate between that of the pure PEO 273 and dextran phases. The fit values of $\tau$ increased with increasing volume fraction of the dispersed 274 phase, but at a given dispersed volume fraction $\tau$ was smaller for $\mathrm{P} / \mathrm{D}$ emulsions ( $\left.\phi_{\mathrm{PEO}} \leq 50 \%\right)$ 275 than for $\mathrm{D} / \mathrm{P}$ emulsions $\left(\phi_{\mathrm{PEO}} \geq 60 \%\right)$.
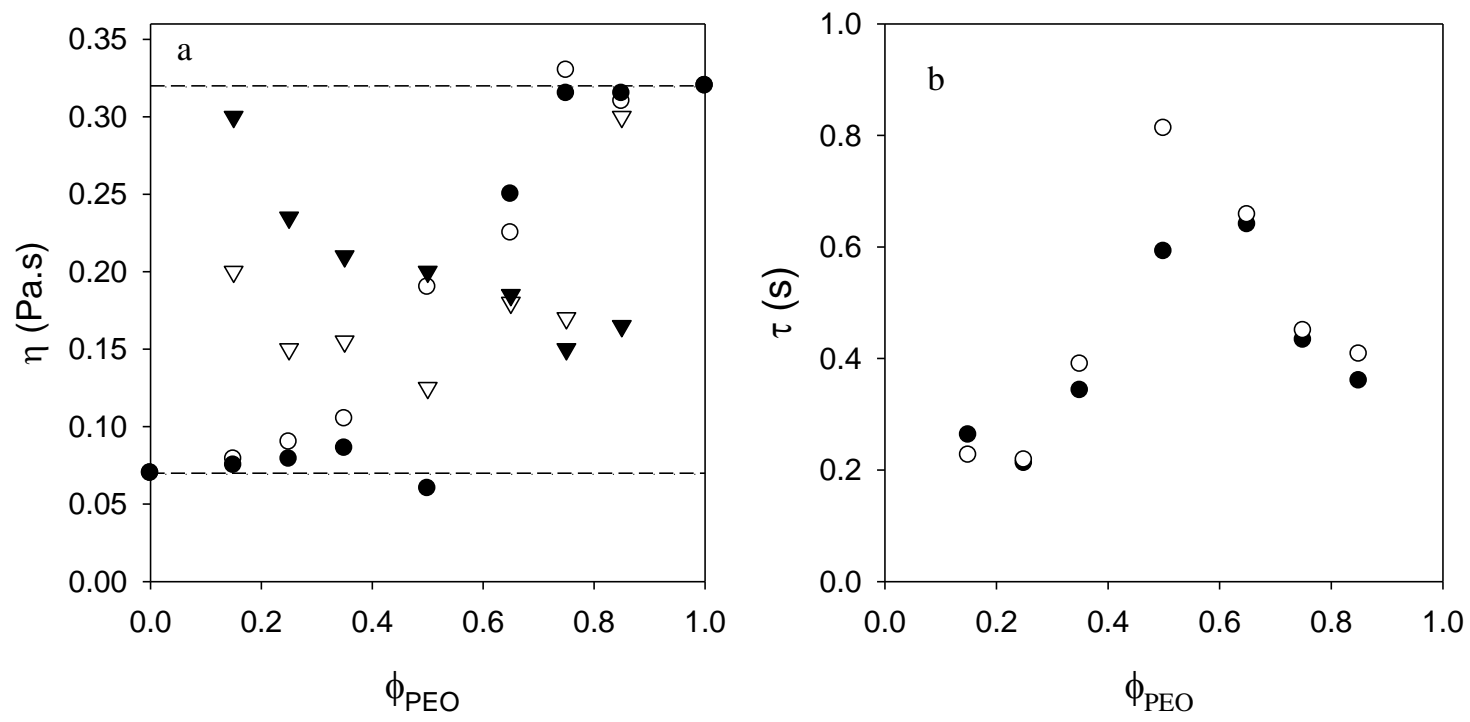

277 Figure 5. Viscosity of the continuous ( $\eta_{c}$, circles) and dispersed ( $\eta_{d}$, triangles) phases (a) and the 278 relaxation time (b) obtained from fits to eq. 2 (filled symbols) and eq. 3 (open symbols). The 
279 dashed lines in figure 5a indicate the viscosities of the pure PEO (upper line) and dextran (lower 280 line) phases.

283 viscosity of the two phases, the interfacial tension and the radius of the droplets, see eq. 4 . The

284 emulsions at different volume fractions were prepared at the same tie-line, which means that 285 only the radius of the droplets varies. The larger $\tau$ at $\phi=35 \%$ corresponds to larger droplet sizes 286 seen with CLSM, see fig. 1. However, the values of R calculated from $\tau$ using the measured 287 interfacial tension $\left(\Gamma=55 \pm 5 \mu \mathrm{N} \cdot \mathrm{m}^{-1}\right)$ were larger $(34-55 \mu \mathrm{m})$ than the volume averaged radii 288 derived from the CLSM images $(9-27 \mu \mathrm{m})$. We conclude that there is a significant discrepancy 289 between the values of $\eta_{c}, \eta_{d}$ and $R$ derived from fits to eqs 2 or 3 and the real values determined 290 independently. Clearly, the theoretical equations developed so far do not correctly describe the 291 viscosity of W/W emulsions during shear flow and new models need to be developed that take 292 into consideration that the droplets strongly deform, orient and coalesce into strings.

\section{Morphology}

Figure 6 shows CLSM images of D/P and P/D emulsions that were taken while shearing

296 at different shear rates. A video of an emulsion during shear flow in real time is added as 297 supporting information. With increasing shear rate the droplets become more deformed and align 298 themselves in the flow. As the shear rate is increased further strings were formed. These results 299 are similar to those reported for other $\mathrm{W} / \mathrm{W}$ emulsions reported in the literature ${ }^{14,}{ }^{17}$. The 300 characteristic string morphology was reached within $1 \mathrm{~s}$ after the start of the measurement and 301 remained the same for the duration of the experiment. Unfortunately, the device used here does 
302 not allow long durations of continuous shear. Therefore, we were not able to investigate the long

303 time evolution of the microstructure with time under shear, which was shown by De Hoog and

304 Tromp ${ }^{14}$ to evolve slowly involving formation of thicker strands and eventually bands.

305

$85 \%$
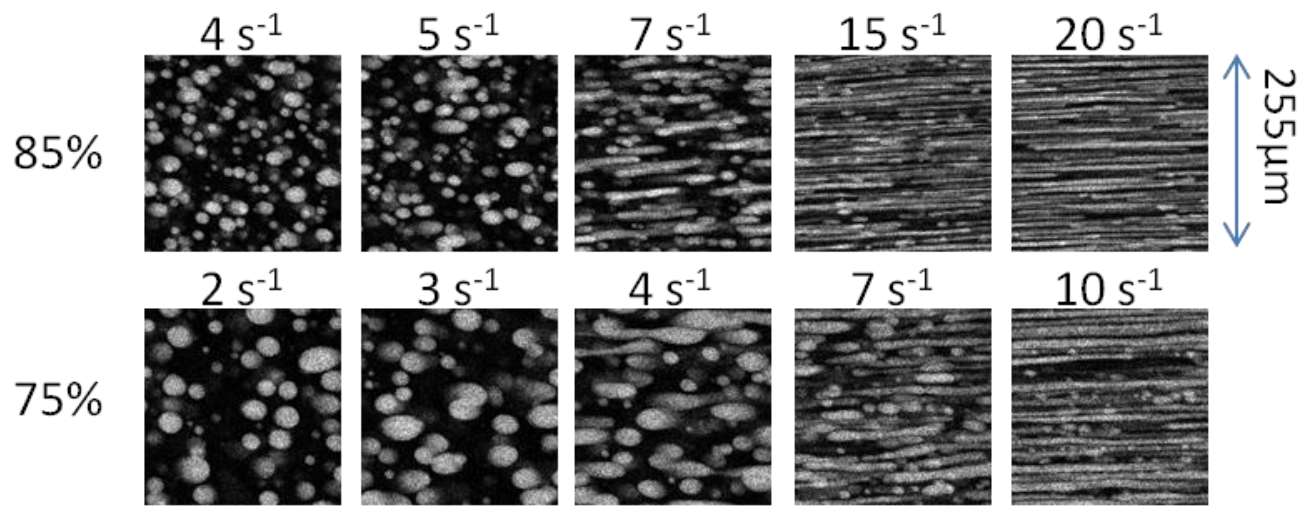

$65 \%$
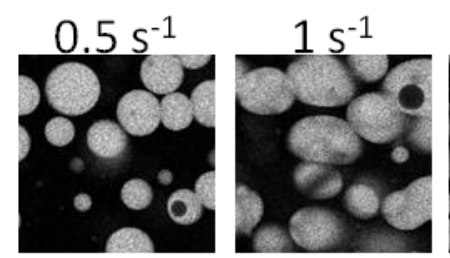

$2 \mathrm{~s}^{-1}$
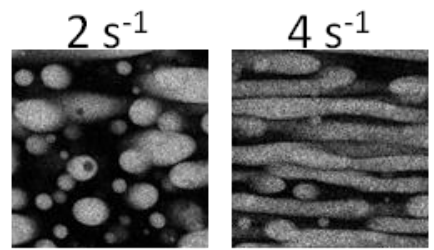

$7 \mathrm{~s}^{-1}$
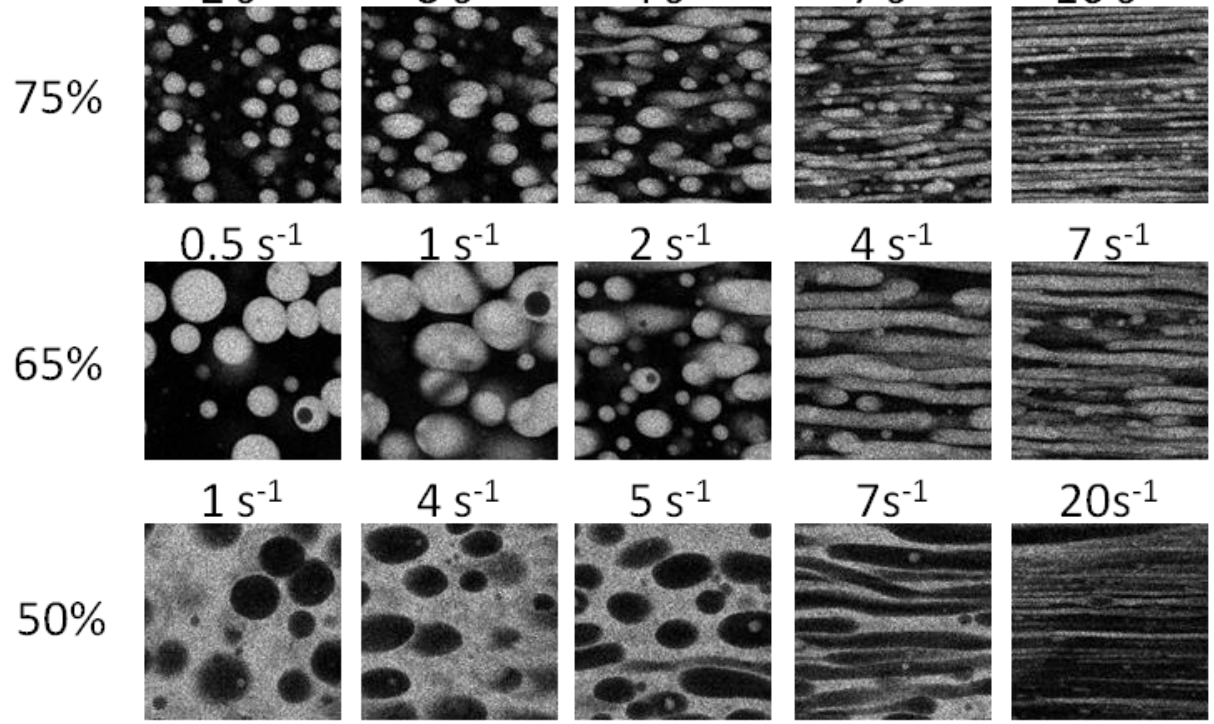

$$
2 \mathrm{~s}^{-1}
$$
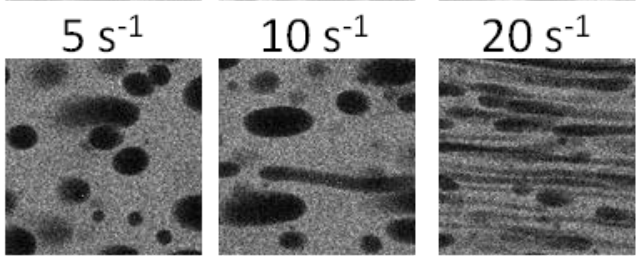

$30 \mathrm{~s}^{-1}$

$35 \%$
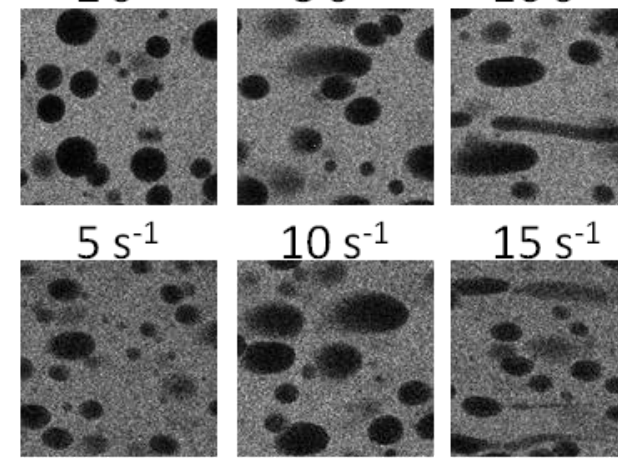

$25 \%$
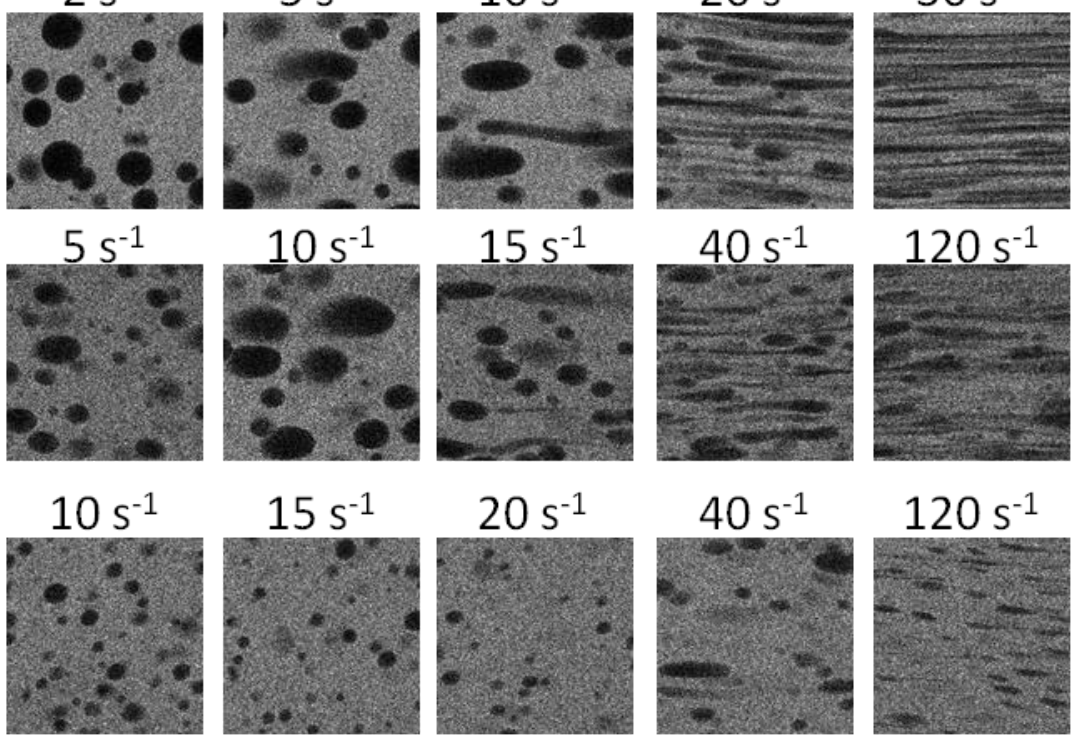

$15 \%$
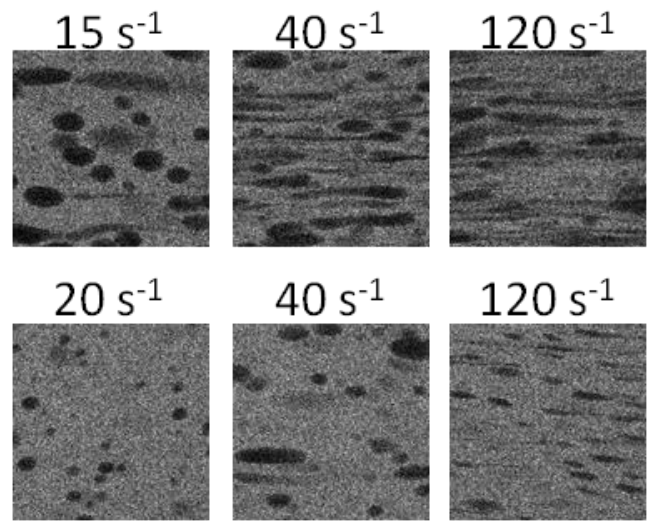
307 Figure 6. CLSM images of emulsions at different phase volume fractions at different shear rates

308 as indicated in the figure. The labeled dextran phase is indicated in white.

With increasing volume fraction of the dispersed phase, the shear rate at which the

311 droplets started to deform decreased, because the droplets at rest were larger. The shear rate at

312 which strings were formed also decreased with increasing $\phi$, indicating that string formation is

313 easier at higher volume fractions. Droplet deformation and string formation started at higher

314 shear rates for $\mathrm{P} / \mathrm{D}$ than for $\mathrm{D} / \mathrm{P}$ emulsions at the same $\phi$ and droplet size, because it is easier to

315 deform droplets that are less viscous than the continuous phase. Comparison with the shear rate

316 dependent viscosity (figure 3) shows that the shear rate at which the droplets started to deform

317 corresponds approximately with the shear rate at which $\eta$ started to decrease. The shear rate at

318 which most droplets were transformed into strings was significantly higher and corresponds

319 approximately to the shear rate at which the second plateau of the viscosity was reached. This

320 correspondence was also noted by Wolf and Frith ${ }^{17}$. They also observed an increase of the

321 critical shear rate with decreasing $\phi$ that was more important when the dispersed phase was more

322 viscous than the continuous phase.

323 Figure 7 shows CLSM images after cessation of the flow. It took approximately one

324 second to arrest all movement. A video is provided as supplementary information. With

325 decreasing shear rate the strands show Rayleigh instabilities and break-up into smaller strands

326 that subsequently contract and become spherical. After cessation of the flow, rapid coalescence

327 occurred between small droplets until approximately the same droplets size distribution as was

328 obtained as before application of the shear. However, since these emulsions were not stabilized,

329 coalescence continued to proceed slowly. We found that this latter process was faster when a low 
330 shear rate was applied, presumably, because it favored collision between droplets. It is clear that

331 the effect of increasing shear rate on the morphology is different from the effect of decreasing

332 shear rate, which explains the hysteresis of the viscosity as a function of the shear rate.

333

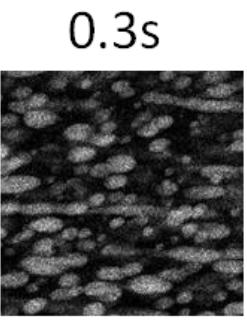

$0.9 \mathrm{~s}$

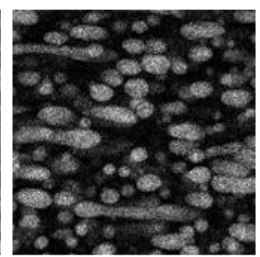

$1.2 \mathrm{~s}$

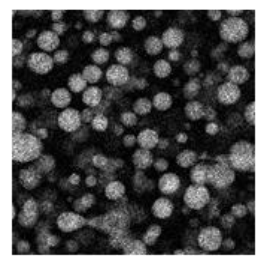

$4.2 \mathrm{~s}$

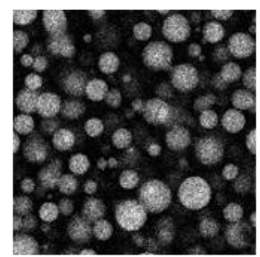

$15 \mathrm{~s}$

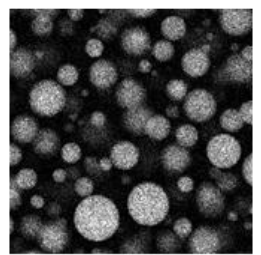

$28 \mathrm{~s}$

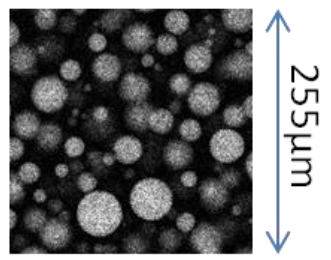

334

335

Figure 7. CLSM Images of a D/P emulsions $\phi=25 \%$ at different times after cessation of the flow at a shear rate of $10 \mathrm{~s}^{-1}$ as indicated in the figure.

\section{Effect of dilution}

$\mathrm{D} / \mathrm{P}$ emulsions at $\phi=25 \%$ were diluted with water. Dextran droplets could still be seen in CLSM images after dilution by a factor 3, but when the emulsions were diluted by a factor 4 they became homogeneous. The volume fractions of the two phases remained the same for dilutions down to a factor two, but $\phi$ was smaller after dilution by a factor $3(\phi=18 \%)$. Figure 8 shows the shear rate dependence of the viscosity at different dilutions. As expected, dilution caused a decrease of the viscosity and the interfacial tension and shifted the effect of shear thinning to higher shear rates. The decrease of the viscosity due to droplet deformation and alignment was observed at approximately the same shear rates down to dilution by a factor 3 , but the effect of hysteresis became smaller and was negligible at dilution x3. Good fits to eq. 2 were obtained with $\tau=0.33 \mathrm{~s}^{-1}$ and $0.34 \mathrm{~s}^{-1}$ for, respectively, dilutions $\mathrm{x} 1.5$ and $\mathrm{x} 2.0$, see solid lines in fig. 8 .

348 The data at dilution $\mathrm{x} 3$ were not good enough to obtained a reliable fit. 


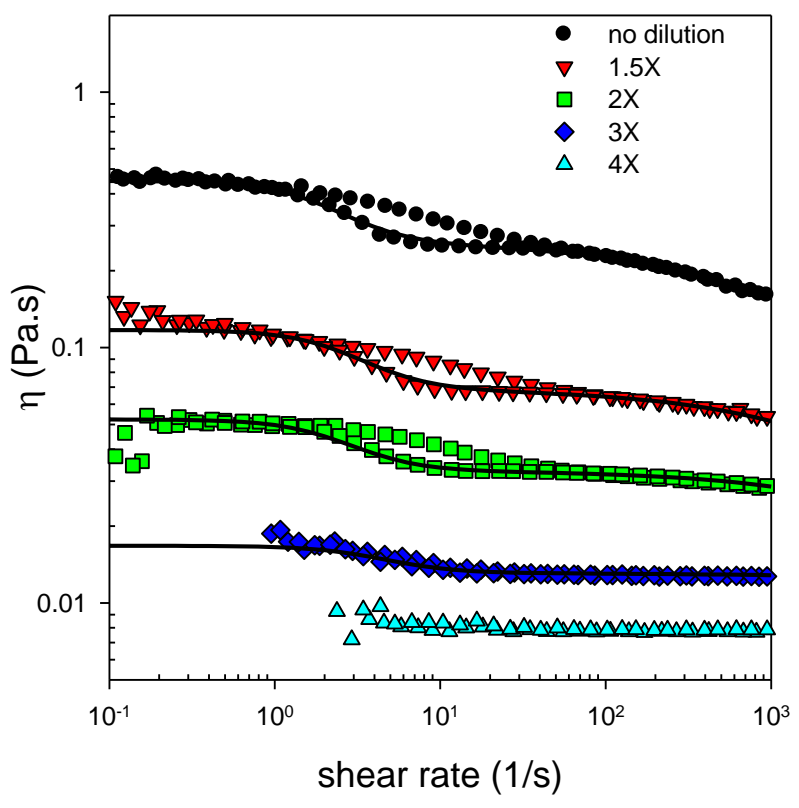

351 Figure 8. Viscosity as a function of increasing shear rate for $\mathrm{D} / \mathrm{P}$ emulsions with $\phi=25 \%$ at 352 different dilutions indicated in the figure. The lines represent fits to eq. 2.

The effect of shear on the morphology did not depend much on the dilution down to $\mathrm{x} 2$, 355 see figure 9 . The droplet size before shear appeared slightly smaller after dilution by a factor 2 356 and the onset of droplet deformation and formation of strands started at slightly lower shear 357 rates. The shear rate at which emulsion droplets start to deform depends on $\mathrm{R}, \mathrm{K}, \Gamma$ and $\eta_{\mathrm{c}}$, see 358 eq. 4, but the effect of dilution on $\mathrm{R}$ and $\mathrm{K}$, which decreases to $\mathrm{K}=2.5$ at dilution $\mathrm{x} 3$, was small 359 compared to the effect on $\Gamma$ and $\eta_{\mathrm{c}}$ which both decreased strongly upon dilution. We found $\Gamma=$ $36016 \pm 2 \mu \mathrm{N} . \mathrm{m}^{-1}, \eta_{\mathrm{c}}=0.09$ Pa.s at dilution $\mathrm{x} 1.5$ and $6 \pm 2 \mu \mathrm{N} . \mathrm{m}^{-1}, \eta_{\mathrm{c}}=0.04$ Pa.s at dilution x2. Using 361 measured values of $\mathrm{K}, \Gamma$ and $\eta_{\mathrm{c}}$, we obtained $\mathrm{R}=30 \mu \mathrm{m}$ and $23 \mu \mathrm{m}$ at dilution $\times 1.5$ and $\times 2$. It 362 appears therefore that, by chance, the decrease of the viscosity is compensated by the decrease of 
363 the interfacial tension, which explains why the shear rate dependence of the viscosity and the 364 morphology was not much influenced by dilution.

a

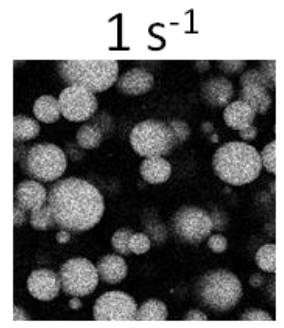

b

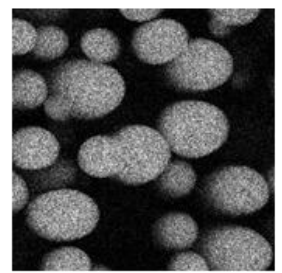

C

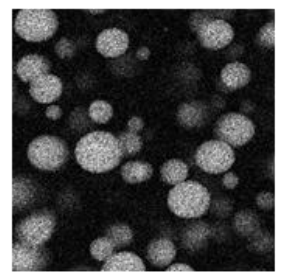

d

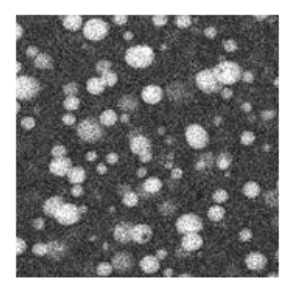

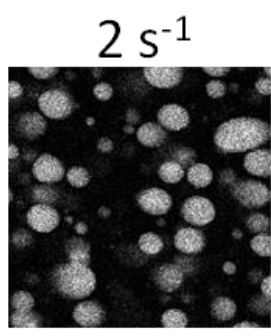
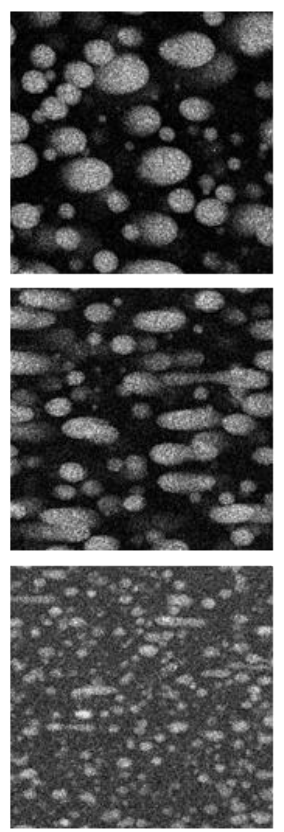
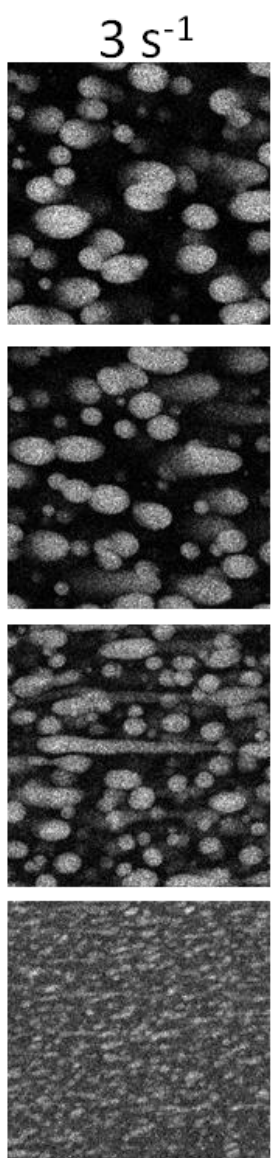

$5 \mathrm{~s}^{-1}$
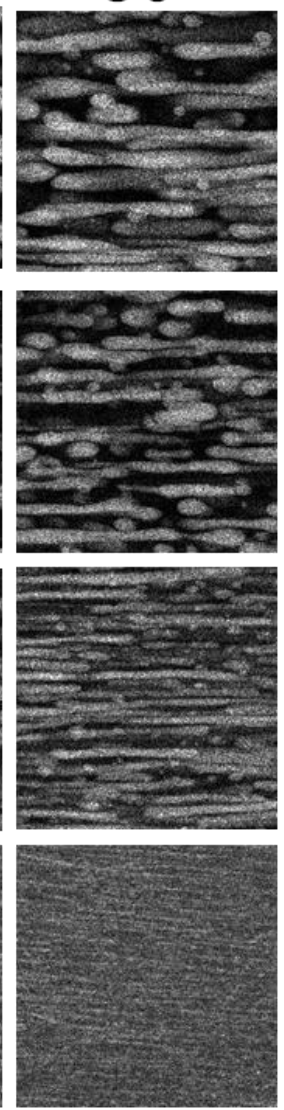

$10 s^{-1}$
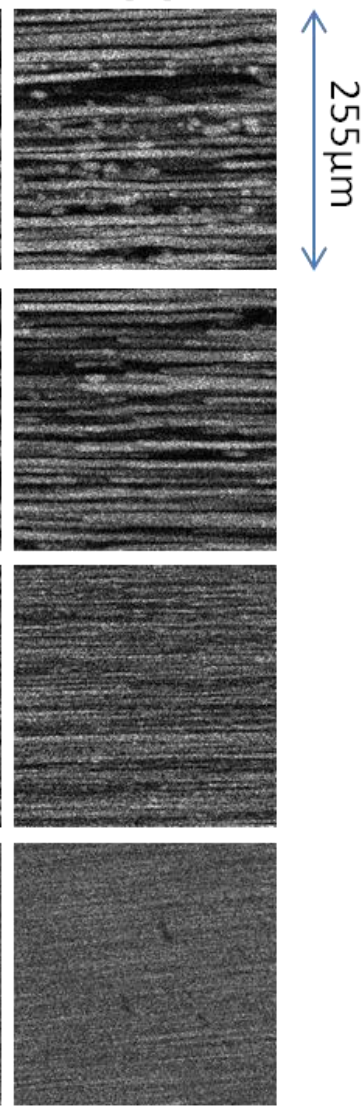

Figure 9. CLSM images of D/P emulsions at $\phi=25 \%$ at different dilutions factors and shear

367 rates. The dilution factors were from top to bottom: x1(a), x1.5(b), x2(c) and x3(d).

\section{Emulsions stabilized by polysaccharides}

D/P emulsions at pH 5 can be stabilized for at least a week by addition of chitosan or

372 DEAED $^{5}$. Interestingly, we found in this study that there was no influence of adding

373 polysaccharides on the interfacial tension within the experimental error. In figure 10 we show the 
374 effect of adding $1 \mathrm{~g} . \mathrm{L}^{-1}$ of chitosan or DEAED on the shear rate dependence of the viscosity of $375 \mathrm{D} / \mathrm{P}$ emulsions with $\phi=25 \%$. The principal effect of adding these polysaccharides was an 376 increase of the viscosity and stronger shear thinning at high shear rates. For comparison, we also 377 show the effect of adding $1 \mathrm{~g} / \mathrm{L}$ k-carrageenan $(\mathrm{KC})$, which is a polysaccharides that does not 378 stabilize $\mathrm{D} / \mathrm{P}$ emulsions. KC had a similar effect on the viscosity as DEAED. CLSM images 379 using fluorescently labeled polysaccharides showed that the majority of the added polymers was 380 distributed between the two phases with only a small amount situated at the interface ${ }^{5}$. The shear 381 rate dependence of the viscosity could be well described by eq. 2 with $\tau=0.31,0.37$ and $0.82 \mathrm{~s}^{-}$ 382 ', for chitosan, DEAED and KC, respectively, see solid lines in fig. 10. The values of $\eta_{\mathrm{c}}$ obtained 383 from the fits were compatible with that of the pure PEO phase containing the amount of added 384 polysaccharide that partitioned to the PEO phase. We note that the viscosity of the 385 polysaccharides in pure water at $1 \mathrm{~g} / \mathrm{L}$ was less than $10^{-2}$ Pa.s. 


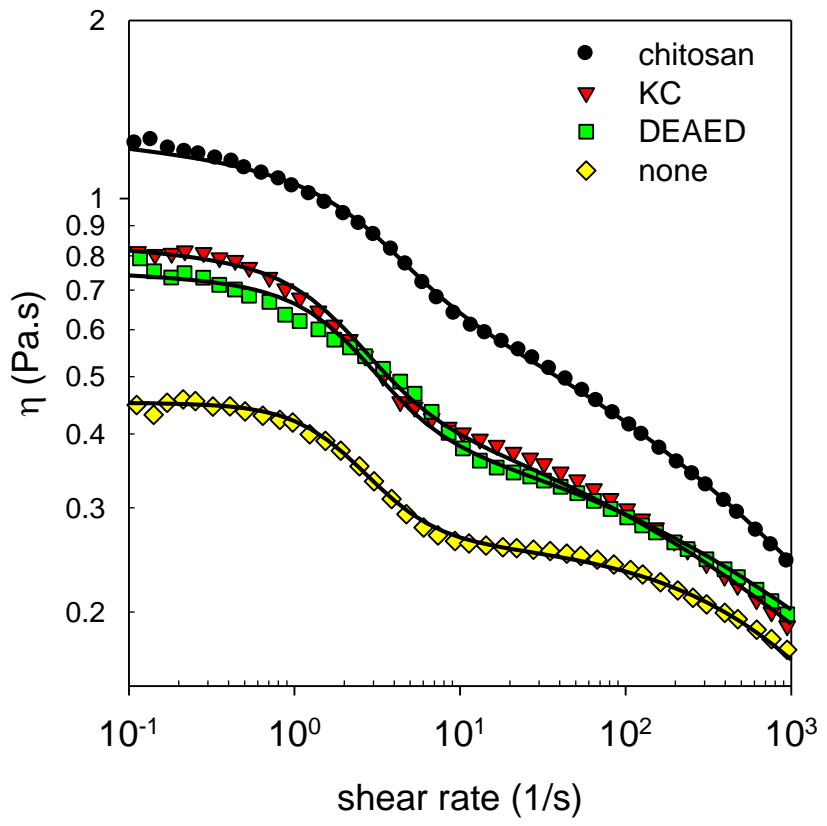

none

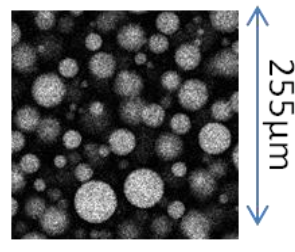

chitosan

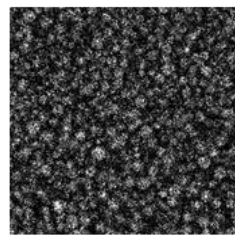

DEAED

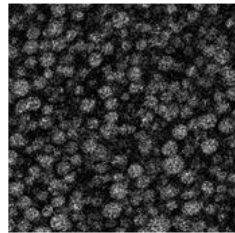

$\mathrm{KC}$

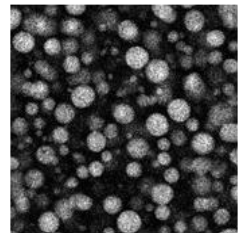

388 Figure 10. Viscosity as a function of increasing shear rate for $\mathrm{D} / \mathrm{P}$ emulsions with $\phi=25 \%$ with

389 or without $1 \mathrm{~g} / \mathrm{L}$ of different added polysaccharides as indicated in the figure. The lines represent

390 fits to eq. 2. CLSM images of the emulsions just after cessation of shear at $10 \mathrm{~s}^{-1}$ are also shown.

CLSM images of the emulsions obtained just after shearing are shown in fig. 10. It is

393 clear that addition of stabilizing polysaccharides has an effect on the droplet size as was already

394 discussed elsewhere ${ }^{5}$. We suggest that the initial rapid coalescence is interrupted when a layer

395 around the droplet is formed that is sufficiently dense and coherent. The size of the droplets

396 immediately after shearing depends therefore on the rate at which the stabilizing layer is formed

397 and not just on the volume fraction of the dispersed phase and the viscosity of the continuous

398 phase. It appears that this process was for these emulsions faste for chitosan than for DEAED 
399 leading to smaller droplets for emulsions containing the former. The rate of subsequent slow 400 coalescence is hugely decreased by the presence of the stabilizing layer.

401 The effect of adding $1 \mathrm{~g} / \mathrm{L}$ of the polysaccharides was also studied at other phase volume 402 fractions, see figures S3 and S4 of the supplementary information. Qualitatively, the effect of the 403 phase volume fractions was similar to that of emulsions without added polymers shown in

404 figures 3 and 5. There are, of course, quantitative differences caused by the higher viscosities of 405 the phases and the smaller droplet size.

406 The effect of adding polysaccharides on the morphology at different shear rates is shown 407 in fig. 11. In all cases strings were formed under high shear. It is clear that the layer of chitosan 408 and DEAED is not strong enough to resist the shear flow even though it does inhibit coalescence 409 at rest. The droplets just after cessation of shear at $10 \mathrm{~s}^{-1}$ were significantly smaller in the 410 presence of chitosan and DEAED. In the presence of $\mathrm{KC}$ the droplets were also smaller than for 411 pure $\mathrm{D} / \mathrm{P}$ emulsions, but larger than in the presence of chitosan or DEAED. The droplets started 412 to align and deform at slightly higher shear rates in the presence of chitosan and DEAED than in 413 the presence of KC. The small effect of chitosan or DEAED on $\tau$ can be explained by a 414 compensation of the increase of the viscosity by the decreases of the droplet size as there was no 415 measurable effect on $\Gamma$. Another important observation is that applying a low shear rate led to 416 more rapid coalescence in the presence of non-stabilizing $\mathrm{KC}$, but not in the presence of 417 stabilizing chitosan and DEAED. 


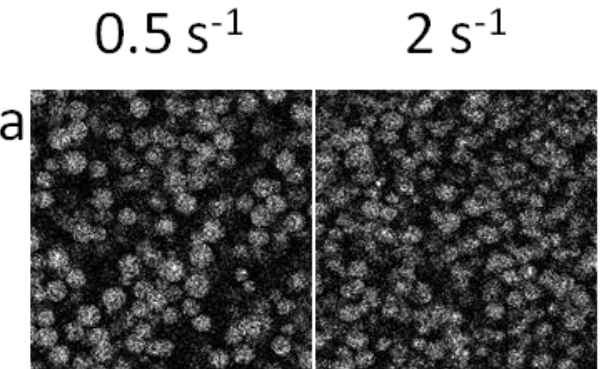

$4 s^{-1}$

$5 s^{-1}$

$10 \mathrm{~s}^{-1}$
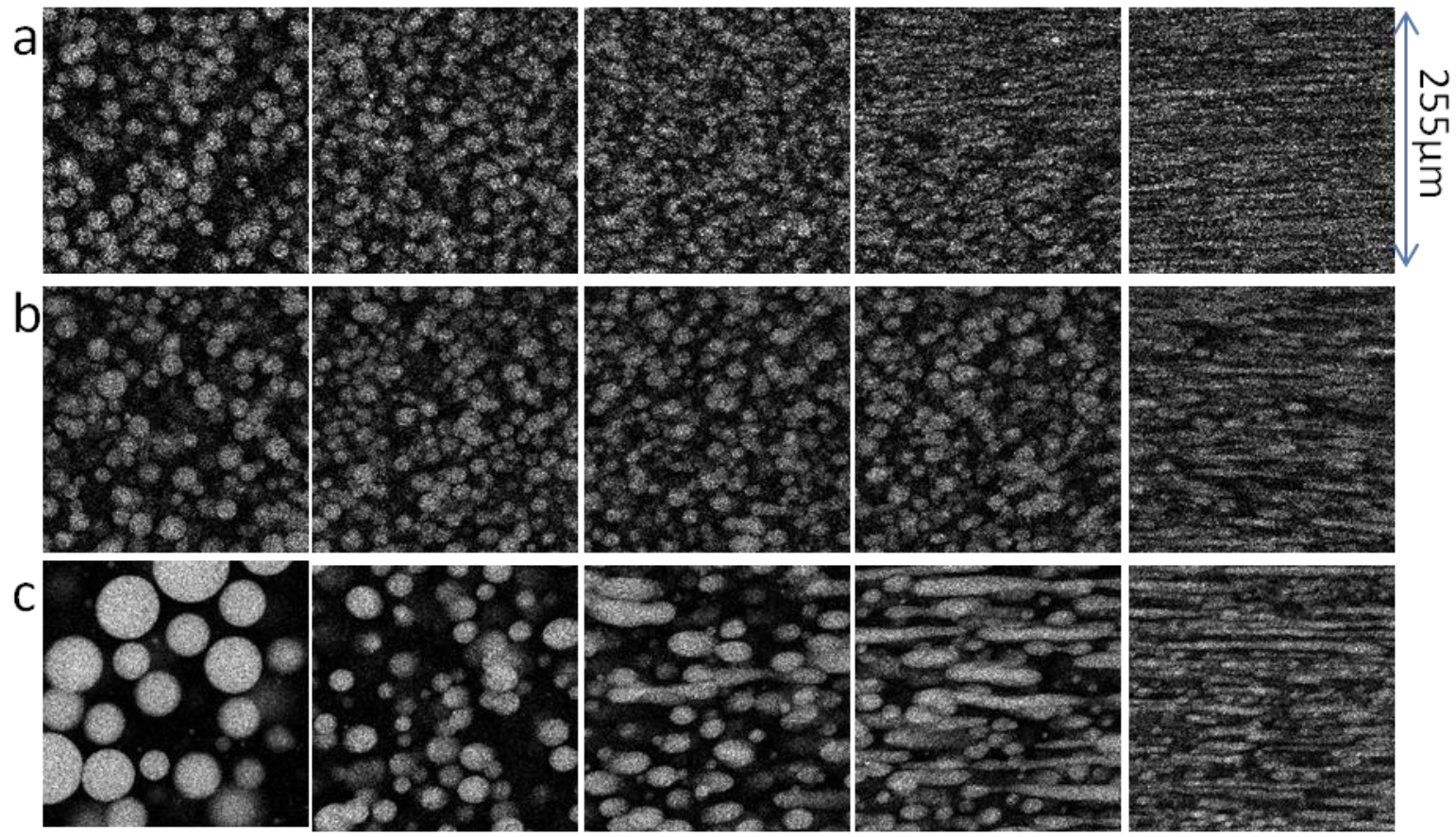

Figure 11. CLSM images of D/P emulsions containing $1 \mathrm{~g} / \mathrm{L}$ chitosan (a), DEAED (b) or KC(c)

421 at different shear rates.

Results obtained with smaller amounts of added polysaccharide were intermediate

424 between those without addition and at $1 \mathrm{~g} / \mathrm{L}$, see figures $\mathrm{S} 5$ and $\mathrm{S} 6$ of the supplementary

425 information. As might be expected, the viscosity and the effect of shear thinning decreased with

426 decreasing chitosan concentration. But $\tau$ obtained from fits to eq. 2 did not depend strongly on

427 the added polysaccharide concentration. CLSM measurements showed that decreasing the

428 chitosan concentration caused an increase of the droplet size after cessation of the shear at $10 \mathrm{~s}^{-1}$,

429 see figure S5. However, there was not a strong effect of the chitosan concentration on the shear

430 rate at which droplet deformation and strand formation started in agreement with the weak

431 variation of $\tau$. The small effect of the added polysaccharide concentration can again be explained 
432 by a compensation between the decrease of the viscosity and the decrease of the droplet size. We

433 may therefore conclude that the effect of adding a stabilizing polymer is principally to inhibit

434 coalescence, but that behaviour of stable and unstable W/W emulsions under shear flow is

435 qualitatively the same.

437 Emulsions stabilized by protein microgels

An important question is whether the particles remain at the interface during shearing and

439 in particular during string formation. The density of the chitosan and DEAED at the interface is

440 low so that it is difficult to visualize the adsorbed layer with CLSM. Therefore, we repeated a

441 few measurements with emulsions in the presence of protein microgels with a radius of about

$442140 \mathrm{~nm}$. A detailed investigation of the effect of adding protein microgels on the stability of

443 these $\mathrm{W} / \mathrm{W}$ emulsions has been reported elsewhere ${ }^{24}$. It was found that the microgels accumulate

444 at the interface both for $\mathrm{D} / \mathrm{P}$ and $\mathrm{P} / \mathrm{D}$ emulsions, but that $\mathrm{P} / \mathrm{D}$ emulsions were more stable than

$445 \mathrm{D} / \mathrm{P}$ emulsions.

446 The shear rate dependence of the viscosity was similar to that observed for pure 447 emulsions or in the presence of polysaccharides, see fig. S8 of the supplementary information.

448 Fig. 12 shows CLSM images of D/P and P/D emulsions at $\phi=25 \%$ containing $1 \mathrm{~g} / \mathrm{L}$ protein 449 microgels. A video is provided as supplementary information. The layer of adsorbed labeled 450 microgels can be clearly seen surrounding the dispersed droplets. We note that excess 451 fluorophore that was not attached to the proteins partitioned preferentially to the PEO phase. 452 With increasing shear, droplet deformation and string formation were observed similar to 453 emulsions in the presence of polysaccharides and started at a higher critical shear rate for the P/D 454 emulsion compared to the $\mathrm{D} / \mathrm{P}$ emulsion. It is clear from the images that the particles remained 
455 adsorbed at the interface even at high shear rates when the strings were very thin. We may

456 assume that the same was the case for the adsorbed chitosan and DEAED.

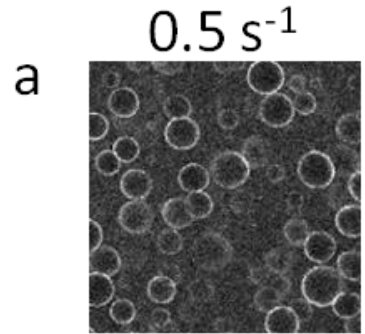

$1 \mathrm{~s}^{-1}$

$\mathrm{b}$

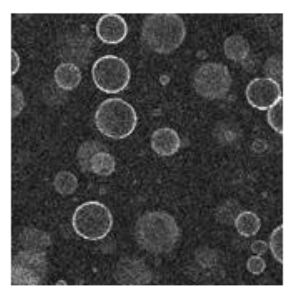

$1 \mathrm{~s}^{-1}$

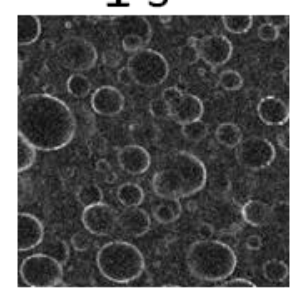

$5 s^{-1}$

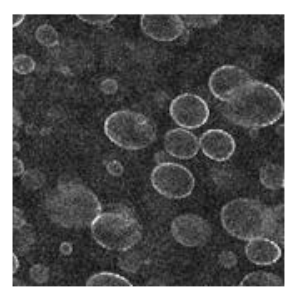

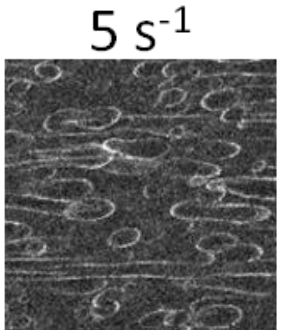

$10 \mathrm{~s}^{-1}$

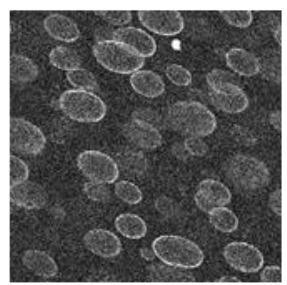

$15 \mathrm{~s}^{-1}$

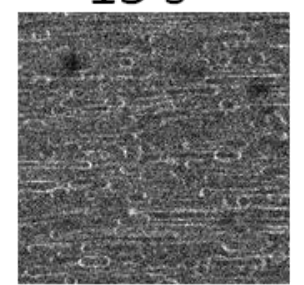

$40 \mathrm{~s}^{-1}$

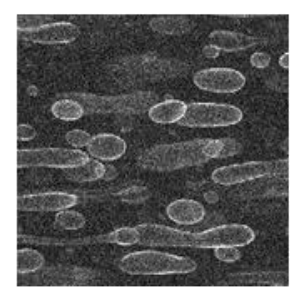

$25 \mathrm{~s}^{-1}$

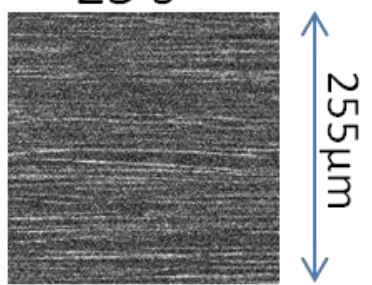

$80 \mathrm{~s}^{-1}$

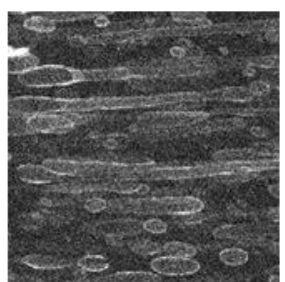

458 Figure 12. CLSM images of D/P (a) and P/D (b) emulsions containing $1 \mathrm{~g} / \mathrm{L}$ of labeled protein

459 microgels at different shear rates. The volume fraction of the dispersed phase was $25 \%$.

Fig. 13 shows the evolution of the morphology of a D/P emulsion after cessation of the

462 flow at a shear rate of $10^{-1}$. The strings rapidly broke up into small droplets that were surrounded

463 by a layer of microgels. Rapid relaxation of deformed droplets and limited coalescence was

464 again observed just after cessation of the flow, but steady state was reached quickly. P/D

465 emulsions remained stable in this state for a period of at least a week, but coalescence continued 466 slowly for D/P emulsions. 

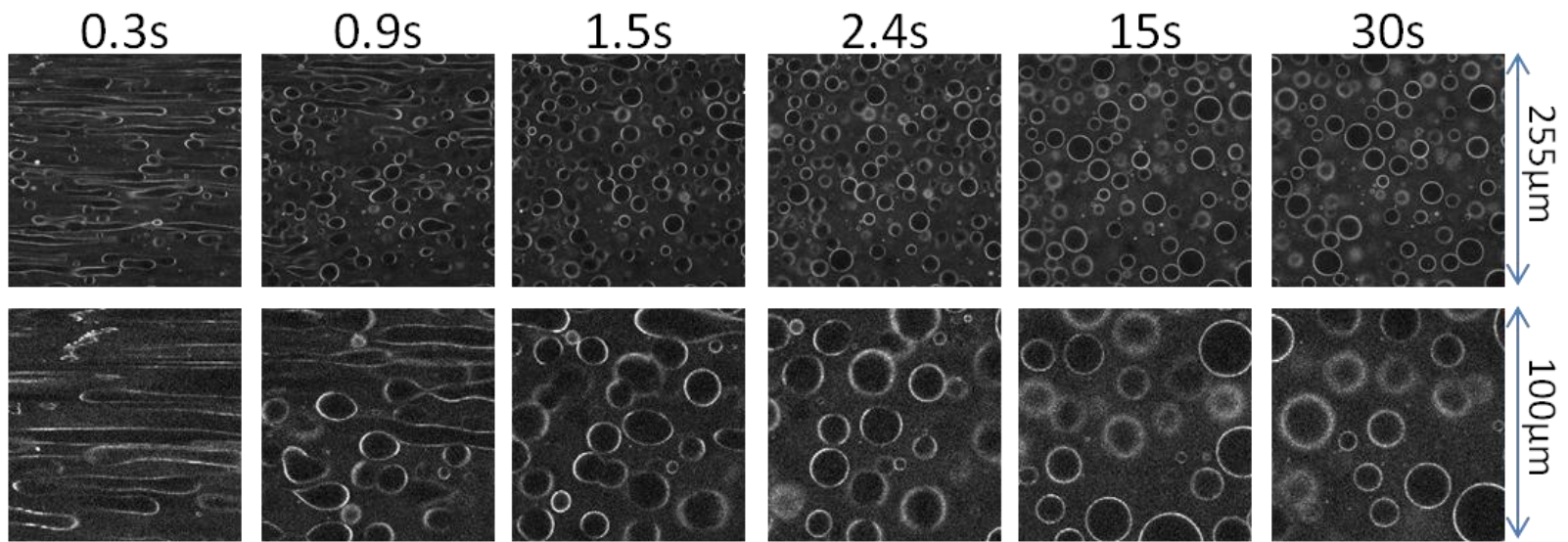

468 Figure 13. CLSM images at two different scales of D/P emulsions containing $1 \mathrm{~g} / \mathrm{L}$ whey protein

469 at different times after cessation of the flow at a rate of $10 \mathrm{~s}^{-1}$ indicated in the figure.

471 Emulsions with the same viscosity for the dispersed and continuous phase.

In this study we have investigated emulsions for which the dispersed phase was either

473 four times more or four times less viscous that the continuous phase. One might wonder whether

474 the difference between in viscosity is important for the behavior of these emulsions under shear

475 even though the equations that were proposed to describe the behavior do not have particular

476 features at $\mathrm{K}=1$. Therefore we prepared $\mathrm{W} / \mathrm{W}$ emulsions with $\eta=0.08 \mathrm{~Pa}$.s for both phases. by

477 choosing a PEO with a lower molar mass $\left(\mathrm{M}_{\mathrm{w}}=1 \times 10^{5} \mathrm{~g} \cdot \mathrm{mol}^{-1}\right)$. Results for emulsions with and

478 without protein microgels showed the same qualitative features both for unstable emulsions and

479 emulsions in the presence of protein microgels, see figures S9-11 of the supplementary

480 information. Strings were still formed with the microgels remaining at the interface. The

481 conclusion is that the general features of the behavior of $\mathrm{W} / \mathrm{W}$ emulsions under shear presented

482 here do not require a significant difference in the viscosity of the two phases.

$484 \quad$ IV. Conclusion 
The viscosity of $\mathrm{W} / \mathrm{W}$ emulsions decreases at a characteristic shear rate due to 487 deformation and alignment of dispersed droplets. At higher shear rates a process of rupture and 488 coalesce leads to the formation of strands that become thinner with increasing shear rate. During 489 cessation of the flow the strands break up into small droplets that rapidly coalesce to form larger 490 droplets and then continue to coalesce slowly. Flow at a low shear rate favors this later stage of 491 slow coalescence.

492 The dependence of the viscosity with increasing shear rate can be well described by 493 equations proposed in the literature if the effect of shear thinning of the two phases is taken into 494 consideration. However, the dependence of the viscosity during decreasing shear rate is different 495 and cannot be described by these equations. At fixed intermediate shear rates the viscosity 496 increased slowly implying that the emulsions evolve with time and that steady state was not 497 reached during the shear rate ramps. The characteristic shear rates where the droplets start to 498 deform and where strings are formed depend on the initial droplet size, the interfacial tension and 499 the viscosities of the two phases.

500 When the W/W emulsions are stabilized by adding certain types of polysaccharides or 501 protein microgels, strand formation is still observed and the behavior under shear flow is not 502 significantly modified by the layer at the interface formed by these particles. However, 503 coalescence of the droplets formed by break-up of the strands after cessation of the shear is 504 inhibited. Particles adsorbed at the interface remain at the interface even when fine strings are 505 formed under strong shear. The small droplets formed immediately after cessation of flow 506 already contain a surface layer of adsorbed particles. 
Supporting Information.

509 -Effect of the duration of the shear rate ramp on the dependence of the viscosity of W/W

510 emulsions on the shear rate.

511 -The effect of the phase volume fractions on the shear rate dependence of the viscosity for 512 emulsions in the presence of $1 \mathrm{~g} / \mathrm{L}$ polysaccharide.

513 -Dependence on $\phi_{\mathrm{PEO}}$ of $\eta_{\mathrm{c}}, \eta_{\mathrm{d}}$, and $\tau$ for emulsions in the presence of $1 \mathrm{~g} . \mathrm{L}^{-1}$ polysaccharide.

514 -Viscosity as a function of the shear rate for D/P emulsions with $\phi_{\mathrm{PEO}}=25 \%$ in the presence of 515 different concentrations of polysaccharide and CLSM images of the emulsions taken shortly after 516 cessation of the shear at $10 \mathrm{~s}^{-1}$.

517 - Viscosity of continuous phase and radii of droplets of different systems obtained from fits to 518 eq. 2.

519 - CLSM images obtained at different shear rates for D/P emulsions at $\phi=25 \%$ in the presence of 520 different concentrations of chitosan.

521 - Viscosity as a function of the shear rate for emulsions at $\phi=0.25$ containing $1 \mathrm{~g} / \mathrm{L}$ protein 522 microgels.

523 - CLSM video of a dextran in PEO emulsions during and after shear at $10 \mathrm{~s}^{-1}$. (Video 1)

524 - CLSM video of a dextran in PEO emulsions containing $1 \mathrm{~g} / \mathrm{L}$ protein microgels during and 525 after shear at $10 \mathrm{~s}^{-1}$. (Video 2)

526 -Behaviour under shear of $\mathrm{W} / \mathrm{W}$ emulsions with the same viscosity for the dispersed and 527 continuous phase.

530 AUTHOR INFORMATION

531 Corresponding Author

532 Taco Nicolai: Taco.Nicolai@univ-lemans.fr.

533 References

534 1. Frith, W. J. Mixed biopolymer aqueous solutions-phase behaviour and rheology. 535 Advances in Colloid and Interface Science 2010, 161 (1-2), 48-60. 
538 3. Nicolai, T.; Murray, B. Particle stabilized water in water emulsions. Food Hydrocolloids 2017, 68, 157-163.

5404 4. Dickinson, E. Particle-based stabilization of water-in-water emulsions containing mixed biopolymers. Trends in Food Science \& Technology 2018, 83, 31-40.

542 5. Tea, L.; Nicolai, T.; Renou, F. Stabilization of Water-in-Water Emulsions by Linear 543 Homo-Polyelectrolytes. Langmuir 2019, 35, 9029-9036.

544 6. Taylor, G. I. The viscosity of a fluid containing small drops of another fluid. Proceedings of the Royal Society of London. Series A, Containing Papers of a Mathematical and Physical Character 1932, 138 (834), 41-48.

547 7. Kroy, K.; Capron, I.; Djabourov, M. On the Viscosity of Emulsions. arXiv preprint 548 physics/9911078 1999.

549 8. Frankel, N.; Acrivos, A. The constitutive equation for a dilute emulsion. Journal of Fluid 550 Mechanics 1970, 44 (1), 65-78.

551 9. Grace, H. P. Dispersion phenomena in high viscosity immiscible fluid systems and 552 application of static mixers as dispersion devices in such systems. Chemical Engineering 553 Communications 1982, 14 (3-6), 225-277.

554 10. Hashimoto, T.; Matsuzaka, K.; Moses, E.; Onuki, A. String phase in phase-separating 555 fluids under shear flow. Physical review letters 1995, 74 (1), 126.

11. Jeon, H.; Hobbie, E. K. Shear viscosity of phase-separating polymer blends with viscous asymmetry. Physical Review E 2001, 63 (6), 061403.

560 13. Migler, K. B. String formation in sheared polymer blends: Coalescence, breakup, and 561 finite size effects. Physical review letters 2001, 86 (6), 1023.

562 14. Tromp, R. H.; De Hoog, E. H. Band formation on shearing in phase-separated polymer 563 solutions. Physical Review E 2008, 77 (3), 031503.

564 15. Capron, I.; Costeux, S.; Djabourov, M. Water in water emulsions: phase separation and 565 rheology of biopolymer solutions. Rheologica Acta 2001, 40 (5), 441-456.

16. Wolf, B.; Frith, W. J.; Singleton, S.; Tassieri, M.; Norton, I. T. Shear behaviour of biopolymer suspensions with spheroidal and cylindrical particles. Rheologica Acta 2001, 40 (3), 238-247.

17. Wolf, B.; Frith, W. J. String phase formation in biopolymer aqueous solution blends. Journal of Rheology 2003, 47 (5), 1151-1170.

18. Choi, S. J.; Schowalter, W. Rheological properties of nondilute suspensions of deformable particles. The Physics of Fluids 1975, 18 (4), 420-427.

19. Ahmed, K. F.; Aschi, A.; Nicolai, T. Formation and characterization of chitosan-protein particles with fractal whey protein aggregates. Colloids and Surfaces B: Biointerfaces 2018, 169, 257-264.

20. Heinze, T.; Liebert, T.; Heublein, B.; Hornig, S., Functional polymers based on dextran. In Polysaccharides Ii, Springer: 2006; pp 199-291.

21. Phan-Xuan, T.; Durand, D.; Nicolai, T.; Donato, L.; Schmitt, C.; Bovetto, L. On the crucial importance of the $\mathrm{pH}$ for the formation and self-stabilization of protein microgels and strands. Langmuir 2011, 27 (24), 15092-15101. 
581 22. Tromp, R.; Blokhuis, E. Tension, rigidity, and preferential curvature of interfaces 582 between coexisting polymer solutions. Macromolecules 2013, 46 (9), 3639-3647.

583 23. Boitte, J.-B.; Vizcaino, C.; Benyahia, L.; Herry, J.-M.; Michon, C.; Hayert, M. In A novel 584 rheo-optical device for studying food complex systems under controlled double plate shear, 585 Review of Scientific Instruments 2013, 84, 013709 (2013).

586 24. Nguyen, B. T.; Nicolai, T.; Benyahia, L. Stabilization of water-in-water emulsions by 587 addition of protein particles. Langmuir 2013, 29 (34), 10658-10664. 